\title{
Efficacy of Implementing Home Care Using Eye Movement Desensitization and Reprocessing in Reducing Stress of Patients with Gastrointestinal Cancer
}

\author{
Milad Borji ${ }^{1}$, Asma Tarjoman², Alireza Abdi', Masoume Otaghi*
}

\begin{abstract}
Background: Gastrointestinal cancer is the third most common types of cancer in the world which leads to a lot of stress among sufferers. Pharmacological and non-pharmacological approaches are used to treat stress induced by serious diseases. Eye movement desensitization and reprocessing (EMDR) technique is considered as one of non-pharmacological method for decreasing patient's stress. Objective: This study was conducted to determine the effect of home care using EMDR technique on the stress of patients with gastrointestinal cancer. Materials and Methods: The current semi-experimental study was performed on patients with gastrointestinal cancer residing in Ilam, Iran. The patients were randomly divided into two groups of intervention $(n=30)$ and control $(n=30)$. Home care was provided for intervention group in patients' homes which included 2 sessions (a total of 60 sessions for all patients). Each session lasted for 45 to 60 minutes according to EMDR protocol. The data were analyzed using SPSS (version 16). Results: The findings of this study showed that most of patients were male $(36,60 \%)$, had diploma degrees $(44,73.3 \%)$, had a monthly income less than 500 thousand $(38,63.3 \%)$, were married $(39,65 \%)$. The mean age of the patients was $69.18 \pm 11.58$ years. No statistically significant difference was observed between two groups before the intervention in terms of patients' perceived stress $(\mathrm{P}>0.05)$. However, efficacy and perceived distress of the intervention group significantly was decreased following the intervention $(\mathrm{P}<0.05)$. Conclusions: According to the findings regarding the impact of home care using EMDR technique on reducing stress in patients with gastrointestinal cancer, the implementation of this intervention and provision of education for patients are recommended to expand the nursing duty to community health wards as well as to improve the health status of patients.
\end{abstract}

Keywords: Eye movement desensitization and reprocessing- cancer- home care

Asian Pac J Cancer Prev, 20 (7), 1967-1971

\section{Introduction}

Cancer is a chronic disease with a growing prevalence and relapse rate (Yuan et al., 2017; Talebi et al., 2018; Khoshnood et al., 2018a; Khoshnood et al., 2018b). Currently, about $12 \%$ of deaths in the world are caused by cancer and annually about 9 million new cases are afflicted with cancer, in which 4 million are from developed countries and 5 million from developing countries (Jemal et al., 2011). In this century, the prevalence of cancer has increased and published statistics show that, among types of cancers, gastrointestinal cancer is the most common type (Menbari et al., 2017; Izadi et al., 2016; Pietrzyk et al., 2016). Gastrointestinal cancer is the third most common type of cancer in the world and its prevalence rates in men and women are 20.6 and 14.3 per hundred thousand people, respectively (Nikbakht et al., 2015; Tahmasbi et al., 2018). Following the detection of cancer in patients, side effects such as crisis, disruption of personal relationships, low self-confidence, lack of adjustment mechanisms, and repeated hospitalization will occur, resulting in psychological stress in patients which can increase the risk of depression in them (Mardani Hamoleh et al., 2011). In fact, chronic diseases place enormous demands on the sufferers. Fulfilling these demands will not be possible without the help and support of a treatment team (Mahmoodishan et al., 2010; Mojen et al., 2018).

To treat stress, several pharmacological and non-pharmacological methods are used. Using pharmacological treatments leads to many side effects; for example, use of antipsychotic drugs, anti-anxiety drugs, and antidepressants can cause cardiac arrhythmias and even sudden death. In addition, pharmaceutical treatments lead to drug resistance and affiliation in patients over time and also will result in huge national costs (Cline et al., 2008). Among the commonly accepted drugs,

${ }^{1}$ Department of Nursing, Faculty of Nursing and Midwifery, ${ }^{2}$ Student Research Committee, Kermanshah University of Medical Science, Kermanshah, ${ }^{3}$ Department of Nursing, Faculty of Nursing and Midwifery, Ilam University of Medical Science, Ilam, Iran. *For Correspondence: otaghi-m@medilam.ac.ir 
Benzodiazepines, which are used for treating anxiety, have serious negative effects such as hypotension, drowsiness, dizziness, loss of balance, cognitive disorders, giddiness, and squint (Imanishi et al., 2009). Due to the high cost of pharmaceutical methods, non-pharmacological methods can be used for controlling stress among patients (Bagherinesami et al., 2003; Hatefi et al., 2015).

Two of the non-pharmacological treatments for anxiety are cognitive-behavioral techniques and eye movement desensitization and reprocessing (EMDR). EMDR is a non-invasive, safe, and simple technique and does not lead to negative effects; in addition, it is not dependent on the medical treatment. Regular and quick movements of the patient's eyes are used in this technique (Rahimi et al., 2016). In EMDR technique, social health nurses ask the patients to remember uncomfortable memories when moving their eyes according to a systematic protocol. As a result, the excitation level is reduced and the patient's thoughts are organized once again (McGuire et al., 2014).

Social health nurses play the most important role in providing nursing services at home, which are provided in many countries. Social health nurses can play an important role in implementing plans regarding health, care, education, and rehabilitation of patients through providing home nursing care services and analyzing and identifying problems of patients (Fallahi-Khoshknab, 2007; Cohen et al., 1983; Borji, 2017). Moreover, the increasing cost of hospitalization, risk of hospital infections, and being away from the family have caused home care nursing to be more popular.

\section{Objectives}

Considering the importance of nursing care at home and given that no research has been conducted on the efficacy of EMDR, this study was conducted to determine the effect of home care using EMDR on the stress of patients with gastrointestinal cancer residing in Ilam, Iran.

\section{Materials and Methods}

The current semi-experimental study containing an intervention group and a control group was performed on patients with gastrointestinal cancer (stomach and colon) who were living in Ilam. Statistical population of this study included 74 patients who were invited to participate in the study, of whom only 65 consented to participate and 5 of them attended only in one of the intervention session. The inclusion criteria were having gastrointestinal cancer (stomach and colon) for at least 6 months since the cancer diagnosis, non-existence of any crisis during the last 6 months, residing in Ilam, and willing to participate in the intervention. Exclusion criteria included the absence in one or more intervention sessions, patient's refusal to participate in the study, and exposure to a crisis during the last three months (including death of a beloved person or undergoing an operation). The data collection tools used included the Perceived Stress Scale developed by Cohen et al., (1983) which contains 14 questions in two sections of perceived self-efficacy (8 questions) and perceived inability (6 questions) (Safaei and Shokri, 2014). To measure respondents' opinions, 4-point Likert scale is used in this questionnaire (never (zero point) to always (four points)). The scores ranged from 0 to 56. In this questionnaire, items 4,5,6,7,9,10, and 13 are reverse scored and the cutoff score is 21.8 , such that higher scores indicate greater perceived stress (Safaei and Shokri, 2014).

In this study, patients were assigned into two groups of intervention and control. Patients were selected from the clients referred for chemotherapy to Shahid Mostafa Khomeini hospital using convenient sampling method and the cases reported by social health nurses randomly assigned to the experimental and control groups by giving them cards in which the name of test or control groups was written. The control group included patients receiving routine care at home by their families. For patients in the intervention group, two intervention sessions were conducted by social health nurses in patients' homes. Intervention sessions were focused on EMDR therapy. EMDR consisted of eight levels that are performed within 45 to 60 minutesv (Rahimi et al., 2016) (Table 1).

Ethical considerations that were observed in this study included: obtaining permission from Ethics Committee of Kermanshah University (IR.KUMS.REC.1397.981), obtaining informed consent from patients, compliance with the provisions of the Declaration of Helsinki, imposing no costs on the patient, random assignment of patients to intervention and control groups, allowing patients to withdraw from the study at any time, providing the necessary training to the control group after the study, and registration of the study at Center for Clinical Trials. The patients participating in this study were assured that their information will be completely confidential. All data were analyzed using SPSS (version 16) and running independent $\mathrm{T}$ test and analysis of variance (ANOVA). $\mathrm{P}<0.05$ was set as the significance level.

\section{Results}

Most patients participating in this study were male (36, 60\%), had diploma degrees $(44,73.3 \%)$, had less than 500 thousand income per month $(38,63.3 \%)$, were married $(39,65 \%)$, and the mean age of patients was $69.18 \pm 11.58$ years. Before conducting the intervention, no statistically significant difference was observed between intervention and control groups in term of demographic variables (Table 2).

According to Table 3, no significant difference regarding perceived stress was observed between two groups before the intervention $(\mathrm{P}>0.05)$. However, after the intervention, patients' perceived stress in the intervention group was significantly decreased $(\mathrm{P}<0.05)$.

\section{Discussion}

Stress affects the health status (Shohani et al, 2018). One of the Role of nurses is helping to improve the health of patients (Shohani et al., 2018 ; Zamanzadeh et al., 2014).

The incidence of cancer is on the rise (Shahrbabaki et al., 2012; Khazaei et al., 2017; Safizadeh et al., 2018). The findings of this study showed that the implementation of 
Table 1. Steps of the Nursing Care at Home Using EMDR Therapy

\begin{tabular}{|c|c|}
\hline Steps & Interventions \\
\hline First step & $\begin{array}{l}\text { Coordination with the patient for nursing care at home, recording the history and description of patient's status, } \\
\text { designing treatment for patient, planning and evaluating the intervention for the patient }\end{array}$ \\
\hline Second step & $\begin{array}{l}\text { Using EMDR therapy, initial stimulation of memory (the patient will be asked to visualize one scene related to } \\
\text { cancer that his mind) }\end{array}$ \\
\hline Third step & $\begin{array}{l}\text { Evoking a secure environment, image, or memory in which patients feel relaxed and then focusing on the } \\
\text { unpleasant feeling }\end{array}$ \\
\hline Forth step & Desensitization of the patient's excitation level \\
\hline Fifth step & Focusing on patient rehabilitation and cognitive reprocessing \\
\hline Sixth step & Physical scanning and evaluation of psychological stress and their assessment \\
\hline Seventh step & Ensuring the stability of the patient \\
\hline Eighth step & Re-evaluating of the treatment effects \\
\hline
\end{tabular}

Table 2. Demographic Data of the Intervention and Control Group Patients

\begin{tabular}{|c|c|c|c|c|}
\hline \multirow[t]{2}{*}{ Demographic variables } & \multirow[t]{2}{*}{ Variable } & \multicolumn{2}{|c|}{ N (\%) } & \multirow[t]{2}{*}{ p-value } \\
\hline & & Experimental & Control & \\
\hline \multirow[t]{2}{*}{ Gender } & Male & $18(60.0)$ & $18(60.0)$ & 1.00 \\
\hline & Female & $12(40.0)$ & $12(40.0)$ & \\
\hline \multirow[t]{2}{*}{ Marital status } & Single & $11(36.7)$ & $10(33.3)$ & 0.79 \\
\hline & Married & $19(63.7)$ & $20(66.7)$ & \\
\hline \multirow[t]{2}{*}{ Education } & Diploma and low literate & $21(70.0)$ & $23(76.7)$ & 0.56 \\
\hline & Collegiate & $9(30.0)$ & $7(23.3)$ & \\
\hline \multirow[t]{3}{*}{ The income per month } & Less than 500 thousand Rials & $20(66.7)$ & $18(60.0)$ & 0.72 \\
\hline & 500 to 1 million & $6(20.0)$ & $8(26.7)$ & \\
\hline & More than 1 million & $4(13.3)$ & $4(13.3)$ & \\
\hline \multirow[t]{3}{*}{ Family support } & Low & $19(63.3)$ & $4(13.3)$ & 0.73 \\
\hline & Mean & $7(23.3)$ & $17(56.7)$ & \\
\hline & alot & $4(13.3)$ & $9(30.0)$ & \\
\hline \multirow[t]{2}{*}{ Drug addiction } & Yes & $18(60.0)$ & $20(66.7)$ & 0.59 \\
\hline & No & $12(40.0)$ & $10(33.3)$ & \\
\hline $\operatorname{Age}(\mathrm{M} \pm \mathrm{SD})$ & & $70.03(11.42)$ & $68.33(11.83)$ & 0.57 \\
\hline
\end{tabular}

home care using EMDR therapy had contributed to the reduction of patients' stress. These results are consistent with those reported by Rahimi et al., (2016), indicating that regular implementation of EMDR technique reduced stress in patients being treated with hemodialysis. One difference between this study and aforementioned study is the location of the research; while EMDR technique was performed at home and under the supervision of social health nurses in the current study, , this technique was performed in a dialysis unit of the hospital in Rahimi et al., (2016)'s study. Moradpur et al., (2014) also used EMDR technique and reported reduced post-traumatic

Table 3. Comparison of the Mean and Standard Deviation of Perceivead Stress of Patients before and after the Intervention

\begin{tabular}{llccc}
\hline Variable & & Before intervention & After intervention & P-value \\
\hline Perceived self-efficacy & Intervention Group & M (SD) & M (SD) & \\
& Control Group & $27.23(4.19)$ & $19.86(5.53)$ & 0.001 \\
& P-value & $0.93(3.94)$ & $27(3.86)$ & \\
Perceived distress & Intervention Group & $20.46(3.40)$ & $15.76(3.88)$ & 0.001 \\
& Control Group & $19.60(3.28)$ & $19.56(3.14)$ & \\
Perceived Stress & P-value & 0.70 & 0.001 & 0.001 \\
& Intervention Group & $47.70(7.58)$ & $35.63(8.52)$ & \\
& Control Group & $46.73(6.15)$ & $47.56(6.04)$ & 0.001 \\
\hline
\end{tabular}


stress in soldiers (Bisson et al., 2007). Additionally, they revealed that EMDR technique reduced symptoms of posttraumatic stress disorders which are compatible with the findings of the current study (Bisson et al., 2007; Davidson and Parker, 2001; Bradley et al., 2005).

The findings of this study are in line with those from other studies suggesting that EMDR technique has a positive effect on patient's stress; for example, studies done by Carletto et al., (2016) on stress in patients with multiple sclerosis, Kratzer et al., (2017) on stress in children and adolescent with post-traumatic stress disorder, and by Schneider et al., (2005) on stress in patients with epilepsy. In order to explain the consistent findings of the current study and the above mentioned results, it can be said that EMDR is a kind of psychotherapeutic technique which includes exposure therapy and cognitive therapy using hearing induction, eye movements, and hand tapping. This technique facilitates the processing of emotional information in the brain and releasing of the stress of patients (Chemtob et al., 2000).

With regards to home care interventions for maintaining the patients' health status, we can refer to a study conducted by Tinetti et al., (2012) demonstrating that the implementation of home care reduced the rate of hospitalization, improved the performance of patients, and reduced the number of referrals to the emergency room. on the other hand, Parsons et al., (2013) showed that the implementation of home care increased physical activity of the elderly, but this intervention did not increase their perceived social protection.

The researcher did not find a study exploring the effect of home care using EMDR technique for patients with cancer, which made the comparison of our findings with those of other studies impossible. The innovation of this research can be noted as the strength of this research, as according to the researcher's, observation there was no study that has evaluated this technique used by social health nurses in homes care. The limitation of this study was using questionnaire which made it impossible for the researcher to verify the information.

In conclusion, regarding the effectiveness of home care using EMDR technique by social health nurse and its positive impact on stress in patients with gastrointestinal cancer, we found that the implementation of this technique by social health nurses can be useful for patients with gastrointestinal cancer.

\section{Acknowledgements}

Student Research Committee, Kermanshah University of Medical Sciences, Kermanshah, Iran (Grant Number: 3007358).

\section{References}

Bagherinesami M, Mohammadi E, Sadeghi R (2003). Effect of Benson relaxation on the rate of disease process in rheumatoid patients referring to rheumatology research center of Imam Khomeini hospital in Tehran in 2000-2001. J MazandaranUniv Med Sci, 13, 22-8.

Bisson M, Jonathan I, Ehlers A, Matthews R (2007).
Psychological treatments for chronic post-traumatic stress disorder: Systematic review and meta-analysis. $\mathrm{Br} J$ Psychiatry, 190, 97-104.

Borji M (2017). Investigating the effect of home care on death anxiety in patients with gastrointestinal cancer. Govaresh, 22, 131-2.

Bradley R, Greene J, Russ E, Dutra L (2005). A multidimensional meta-analysis of psychotherapy for PTSD. Am J Psychiatry, 162, 214-27.

Carletto S, Borghi M, Bertino G, et al (2016). Treating post-traumatic stress disorder in patients with multiple sclerosis: a randomized controlled trial comparing the efficacy of eye movement desensitization and reprocessing and relaxation therapy. Front Psychol, 7, 526.

Chemtob CM, Tolin D, van K, Bessel A (2000). Eye movement desensitization and reprocessing. (30 January 2010), https:// doi.org/10.1002/9780470479216.corpsy9780470470337.

Cline M, Taylor J, Flores J, Bracken S, McCall S (2008). Investigation of the anxiolytic effects of linalool, a lavender extract, in the male Sprague-Dawley rat. AANA J, $\mathbf{7 6 .}$

Cohen S, Kamarck T, Mermelstein R (1983). A global measure of perceived stress. J Health Soc Behav, 10, 385-96.

Davidson P, Parker K (2001). Eye movement desensitization and reprocessing (EMDR): a meta-analysis. J Consult Clin Psychol, 69, 305

Fallahi-Khoshknab M (2007). Effectiveness of psychiatric nursing home care services for psychiatric status in schizophrenics. Arch Med Rehabil, 8, 77-81.

Hatefi M, Jaafarpour M, Khani A, Khajavikhan J (2015). The effect of whole body massage on the process and physiological outcome of trauma ICU patients: a double-blind randomized clinical trial. J Clin Diagn Res, 9, UC05.

Imanishi J, Kuriyama H, Shigemori I, et al (2009). Anxiolytic effect of aromatherapy massage in patients with breast cancer. Evid Based Complement Alternat Med, 6, 123-8.

Izadi A, Sirizi MJ, Esmaeelpour S, Barouni M (2016). Evaluating direct costs of gastric cancer treatment in Iran-case study in Kerman city in 2015. Asian Pac J Cancer Prev, 17, 3007-13.

Jemal A, Bray F, Center M, Ferlay J, Ward E (2011). Global cancer statistics. CA Cancer J Clin, 61, 69-90.

Khazaei S (2017). Epidemiology of lung cancer in Iran: sex difference and geographical distribution. Middle East $J$ Cancer, 2017, 223-8.

Khoshnood Z, Iranmanesh S, Rayyani M, Dehghan M (2018). Body-mind healing strategies in patients with cancer: a Qualitative content analysis. Asian Pac J Cancer Prev, 19, 1691-6.

Khoshnood Z, Iranmanesh S, Rayyani M, Dehghan M (2018). Getting out or remaining in the cage of inauthentic self: The meaning of existential challenges in patients' with cancer. Indian J Palliat Care, 24, 131.

Mahmoodishan Gh, Alhani F, Ahmadi F (2010). Iranian nurses' perception of spirituality and spiritual care: a qualitative content analysis study. J Med Ethics Hist, 3.

Mardani H, Marjan R, Mahin R (2011). The effect of psycho educational program on stress and depression among cancer patients. J Fasa Univ Med Sci, 1, 53-8.

McGuire M, Lee Ch (2014). Potential of eye movement desensitization and reprocessing therapy in the treatment of post-traumatic stress disorder. Psychol Res Behav Manag, 7, 273.

Menbari M, Nasseri Sh, Menbari N, et al (2017). The-160 (C>A) $\mathrm{CDH} 1$ gene promoter polymorphism and its relationship with survival of patients with gastric cancer in Kurdistan. Asian Pac J Cancer Prev, 18, 1561-5.

Mojen L, Rassouli M, Eshghi P, et al (2018). Pediatric palliative 
care in Iran: Applying regionalization of health care systems. Asian Pac J Cancer Prev, 19, 1303-11.

Nikbakht H, Sani N, Jafarabadi M (2015). Quality of life and its determinants among colorectal cancer survivors. J Kermanshah Univ Med Sci, 19, 84-92.

Parsons J, Geoffrey M, Sheridan N, et al (2013). A randomized controlled trial to determine the effect of a model of restorative home care on physical function and social support among older people. Arch Phys Med Rehabi, 94, 1015-22,

Pietrzyk L, Plewa Z, Denisow-Pietrzyk M, Zebrowski R, Torres K (2016). Diagnostic power of blood parameters as screening markers in gastric cancer patients. Asian Pac J Cancer Prev, 17, 4433-7.

Rahimi F, Heravi-Karimooi NA, Tadrisi, SD (2016). Effect of eye movement desensitization and reprocessing on stress in patients undergoing hemodialysis. Iran J Nurs Res, 11, 1-7.

Safaei M, Shokri O (2014). Assessing stress in cancer patients: Factorial validity of the perceived stress scale in Iran. IJPN, 2, 13-22.

Schneider GN, Darius M, Heuft G (2005). Eye movement desensitization and reprocessing in the treatment of posttraumatic stress disorder in a patient with comorbid epilepsy. Epilepsy Behav, 7, 715-8.

Safizadeh H, Hafezpour F (2018) Health damaged context: Barriers to breast cancer screening from viewpoint of Iranian health volunteers. Asian Pac J Cancer Prev, 19, 1941.

Shahrbabaki PM (2012). The evaluation of the educational plan of breast self-examination of women referring to health centers. Procedia Soc Behav Sci, 31, 913-7.

Shohani M, Abedi L, Rasouli M (2018). Professional attitude in Iranian nursing students. $J C D R, 12$.

Shohani M, Badfar G, Nasirkandy MP, et al (2018). The effect of yoga on stress, anxiety, and depression in women. Int J Prev Med, doi: 10.4103/ijpvm.IJPVM_242_16. eCollection 2018.

Talebi SS, Badfar G, Shohani M, Soleymani A, Azami M (2018). The relationship between selenium and lung cancer: An updated systematic review and meta-analysis. IJCM, 11.

Tinetti E, Charpentier P, Gottschalk M (2012). Effect of a restorative model of posthospital home care on hospital readmissions. J Am Geriatr Soc, 60, 1521-6.

Tahmasbi B, Abedi G, Moosazadeh M, et al (2018). Determining the survival rate of colorectal cancer in Iran: A systematic review and meta-analysis. Asian Pac J Cancer Prev, 19, 3009.

Yuan W, Chen J, Shu Y, et al (2017). Correlation of DAPK1 methylation and the risk of gastrointestinal cancer: A systematic review and meta-analysis. PLoS One, 12, e0184959.

Zamanzadeh V, Irajpour A, Valizadeh L, Shohani M (2014). The meaning of collaboration, from the perspective of Iranian nurses: A qualitative study. Sci World J, doi: 10.1155/2014/785942. Epub 2014;2014:785942.

\section{c) (7) (8)}

This work is licensed under a Creative Commons AttributionNon Commercial 4.0 International License. 